PROCEEDINGS OF THE

AMERICAN MATHEMATICAL SOCIETY

Volume 133, Number 6, Pages 1569-1572

S 0002-9939(05)07618-5

Article electronically published on January 13, 2005

\title{
LIE ALGEBRAS WITH FINITE GELFAND-KIRILLOV DIMENSION
}

\author{
DAVID RILEY AND HAMID USEFI
}

(Communicated by Martin Lorenz)

\begin{abstract}
We prove that every finitely generated Lie algebra containing a nilpotent ideal of class $c$ and finite codimension $n$ has Gelfand-Kirillov dimension at most $\mathrm{cn}$. In particular, finitely generated virtually nilpotent Lie algebras have polynomial growth.
\end{abstract}

\section{Introduction AND STATEMEnt of RESUlts}

Groups with polynomial growth are very well understood. Indeed, according to the famous result of Milnor, Wolf and Gromov (4]), a finitely generated group has polynomial growth precisely when it contains a nilpotent normal subgroup of finite index. While the class of associative algebras with polynomial growth has not yet yielded a similar characterisation, Berele ([3]) has provided us with an illuminating sufficient condition: every finitely generated associative algebra satisfying a nontrivial polynomial identity has polynomial growth.

In contrast, the class of Lie algebras with polynomial growth remains much of a mystery. On the one hand, Lichtman and Ufnarovski ([8]) proved that the growth of every finitely generated free soluble Lie algebra of derived length $k \geq 3$ is almost exponential (and therefore not polynomial). So not every finitely generated Lie PI-algebra has polynomial growth; in other words, the most obvious Lie-theoretic analogue of Berele's theorem is false.

On the other hand, it follows from results of Shmelkin that the case of derived length 2 is correctly omitted since every $n$-generator metabelian Lie algebra has Gelfand-Kirillov dimension at most $n$ (see [11, 7] or [6]). Petrogradsky extended this result in 9] to the class of all finitely generated nilpotent-by-nilpotent Lie algebras ( $c f$. the Corollary below). Our present aim is to extend Petrogradsky's result to the class of all finitely generated virtually nilpotent Lie algebras. Recall that an algebra is said to be virtually in a class $\mathcal{X}$ if it contains an ideal of finite codimension in the class $\mathcal{X}$.

Theorem. Let $L$ be a finitely generated Lie algebra over a field $F$. If $L$ contains a nilpotent ideal $I$ of class $c$ such that $\operatorname{dim}_{F}(L / I)=n<\infty$, then $G K$-dim $(L) \leq c n$. In particular, every finitely generated virtually nilpotent Lie algebra has polynomial growth.

Received by the editors August 27, 2003 and, in revised form, December 9, 2003.

2000 Mathematics Subject Classification. Primary 17B05, 16P90.

The research of the first author was supported by NSERC of Canada. 
In Section 3, we shall provide an example to show that this estimate is precise. Let $\mathcal{N}_{c}$ denote the variety of all nilpotent Lie algebras of class at most $c$.

Corollary ([9]). The free k-generator Lie algebra in the variety $\mathcal{N}_{c} \mathcal{N}_{d}$ has GKdimension at most $c n$, where $n$ is the dimension of the free $k$-generated Lie algebra in $\mathcal{N}_{d}$.

Unlike in the group-theoretic situation, being virtually nilpotent is not a necessary condition for a finitely generated Lie algebra to have polynomial growth. In fact, there exist Lie algebras of GK-dimension 1 that are not even virtually soluble:

Example A. Let $F$ be a field of characteristic zero and denote by $W$ the positive part of the Witt algebra. In other words, $W$ is the Lie $F$-algebra with basis $\left\{e_{i} \mid i \geq\right.$ $1\}$ and relations $\left[e_{i}, e_{j}\right]=(j-i) e_{i+j}$. Direct calculations show that GK-dim $(W)=1$ and yet $W$ is not virtually soluble.

Example B. Let $p$ be a prime and let $F$ be a field of characteristic $p$. Then according to a result of Shalev ([10]), there exists a graded Lie $F$-algebra $L=$ $\bigoplus_{i \geq 1} L_{i}$ of maximal class that is not virtually soluble. Here having maximal class means that $L$ is generated by $L_{1}$ and satisfies $\operatorname{dim}_{F}\left(L_{1}\right)=2$ and $\operatorname{dim}_{F}\left(L_{i}\right)=1$ for all $i \geq 2$. The GK-dimension of any such algebra is 1 .

Other known classes of Lie algebras with polynomial growth include Kac-Moody, Virasoro and Cartan Lie algebras. See [5] for details.

Before closing this section, the authors would like to thank Vesselin Drensky and Alexander Lichtman for their useful comments.

\section{Proof of the Theorem}

Lemma 1. Let L be a finitely generated Lie algebra containing a nilpotent ideal I of finite codimension. Then $I$ is finitely generated as an ideal of $L$.

Proof. We use induction on the nilpotence class $c$ of $I$. In the case $c=1, L$ is finitely generated and virtually abelian; therefore, by a result of Amayo and Stewart (see [1]), every ideal of $L$ is finitely generated. Now suppose $I$ has class $c>1$. Then, by the induction hypothesis, $I / I^{c}$ is finitely generated as an ideal of $L / I^{c}$; in other words, there exists a finite-dimensional subspace $U$ of $I$ such that

$$
I=\sum_{k \geq 0}[U, \underbrace{L, \ldots, L}_{k}]+I^{c} .
$$

Because $I^{c+1}=0$, it follows that $I^{c}$ is contained in the ideal of $L$ generated by $U$. Thus $I$ is generated by $U$, as required.

Henceforth, $L$ is a fixed finitely generated Lie algebra containing a nilpotent ideal $I$ of class $c$ and finite codimension $n$ in $L$. Let $V$ be a vector space complement of $I$ in $L$ with basis $\left\{e_{1}, \ldots, e_{n}\right\}$. For each pair $i, j$, write $\left[e_{i}, e_{j}\right]=x_{i j}+v_{i j}$, where $x_{i j} \in I$ and $v_{i j} \in V$. By the previous lemma, there exists a finite generating set $\left\{x_{1}, \ldots, x_{m}\right\}$ of $I$ that contains each of the $x_{i j}$. Let $U$ be the subspace of $I$ spanned by $\left\{x_{1}, \ldots, x_{m}\right\}$ and set $W=U+V$.

Lemma 2. The subspace $W$ generates $L$ as a Lie algebra. 
Proof. Let $S$ be the subalgebra generated by $W$. Since $L=I+V$, it suffices to show that $I \subseteq S$. Observe then that

$$
I=\sum_{k \geq 0}[U, \underbrace{L, \ldots, L}_{k}]=\sum_{k \geq 0}[U, \underbrace{V, \ldots, V}_{k}]+I^{2} \subseteq S \cap I+I^{2} .
$$

It follows that $I^{r} \subseteq S \cap I+I^{r+1}$ for each $r \geq 1$. But $I^{c+1}=0$ and so the result follows.

Let $W^{(k)}$ denote the subspace of $L$ spanned by all monomials of degree at most $k$ in the generating set $\left\{x_{1}, \ldots, x_{m}, e_{1}, \ldots, e_{n}\right\}$ of $L$. We shall call any monomial of the form

$$
\left[x_{i(1)}, e_{j(1,1)}, \ldots, e_{j\left(1, s_{1}\right)}, x_{i(2)}, e_{j(2,1)}, \ldots, e_{j\left(2, s_{2}\right)}, \ldots, x_{i(t)}, e_{j(t, 1)}, \ldots, e_{j\left(t, s_{t}\right)}\right]
$$

straight if we have $j(r, 1) \leq \cdots \leq j\left(r, s_{r}\right)$ for each $1 \leq r \leq t$. Finally, we shall denote by $I_{k}$ the subspace of $I$ spanned by all straight monomials of total degree at most $k$.

Lemma 3. $W^{(k)} \subseteq I_{k}+V$ for each $k \geq 1$.

Proof. Let $\mu$ be any monomial of degree $r \leq k$ in the $x_{i}$ 's and $e_{j}$ 's. We shall use induction on $r$ to prove $\mu \in I_{k}+V$. The case $r=1$ is trivial. Suppose then that $r>1$ and that $\mu$ is not straight. Then $\mu$ begins (on the left) with a monomial of the form $\mu_{1}=\left[\nu, e_{b}, e_{a}\right]$ where $a<b$. By the Jacobi identity we see that

$$
\mu_{1}=\left[\nu, e_{a}, e_{b}\right]+\left[e_{a}, e_{b}, \nu\right]=\left[\nu, e_{a}, e_{b}\right]-\left[\nu, x_{a b}+v_{a b}\right] .
$$

But $x_{a b}$ is one of the $x_{i}$ 's, and $v_{a b}$ is a linear combination of the $e_{j}$ 's. Hence, $\mu_{1}$ is congruent to a 'straighter' monomial modulo a linear combination of monomials of lesser degree. Appealing to the induction hypothesis, it follows that $\mu$ is congruent modulo $I_{k}+V$ to a straighter monomial of the same degree $r$. Because any monomial will be straightened after a finite series of such transpositions, the lemma is proved.

We are now ready to complete the proof of our theorem.

Lemma 4. The GK-dimension of $L$ is at most cn.

Proof. A simple counting argument followed by crude estimates shows that the number of straight monomials of degree $t$ in the $x_{i}$ 's and total degree $r$ is

$$
m^{t} \sum_{r_{1}+\cdots+r_{t}=r-t} \prod_{i=1}^{t}\left(\begin{array}{c}
r_{i}+n-1 \\
n-1
\end{array}\right) \leq m^{t} r^{t n-1},
$$

and thus

$$
\operatorname{dim}\left(I_{k}\right) \leq \sum_{r=1}^{k} \sum_{t=1}^{c} m^{t} r^{t n-1} \leq c m^{c} k^{c n} .
$$

It now follows from the previous lemma that $\operatorname{dim}\left(W^{(k)}\right) \leq c m^{c} k^{c n}+n$, for each $k \geq 1$. Therefore, GK-dim $(L)=\varlimsup_{k \rightarrow \infty} \log _{k}\left(\operatorname{dim}\left(W^{(k)}\right)\right) \leq c n$. 


\section{A CRITICAL EXAMPLE}

To illustrate that our upper bound is best possible, we adapt Shmelkin's construction of a verbal wreath product (see [2], for example). For each finite-dimensional Lie algebra $L$ with basis $\left\{e_{1}, \ldots, e_{n}\right\}$ consider the free right $L$-module $M$ with generators $\left\{x_{1}, \ldots, x_{c}\right\}$. Then

$$
M_{0}=\left\{x_{i} e_{1}^{a_{1}} \cdots e_{n}^{a_{n}} \mid a_{j} \geq 0,1 \leq i \leq c\right\}
$$

is a basis for $M$. Next consider the free Lie algebra $N$ in $\mathcal{N}_{c}$ on the set of generators $M_{0}$. One can make $N$ into a right $L$-module via $\left[n_{1}, n_{2}\right] g=\left[n_{1} g, n_{2}\right]+\left[n_{1}, n_{2} g\right]$, $n_{1}, n_{2} \in N, g \in L$. The semidirect product $N+L$ is spanned by $\left\{e_{1}, \ldots, e_{n}\right\}$ together with the set of monomials of degree at most $c$ in the generating set $M_{0}$. Furthermore, monomials

$$
\left[x_{1} e_{1}^{a_{1}} \cdots e_{n}^{a_{n}}, x_{2} e_{1}^{b_{1}} \cdots e_{n}^{b_{n}}, \ldots, x_{c} e_{1}^{c_{1}} \cdots e_{n}^{c_{n}}\right]
$$

are linearly independent. The number of such monomials of degree at least $c+1$ and at most $k$ is

$$
\sum_{r=c+1}^{k}\left(\begin{array}{c}
r-c+c n-1 \\
c n-1
\end{array}\right) \geq \sum_{r=c+1}^{k} \frac{(r-c)^{c n-1}}{(c n-1) !} \geq \frac{(k-c)^{c n}}{(c n) !} .
$$

It follows that GK-dim $(N+L) \geq c n$. Consequently, GK-dim $(N+L)=c n$ according to our Theorem.

\section{REFERENCES}

[1] R.K. Amayo and I.N. Stewart, Finitely generated Lie algebras, J. London Math. Soc. 5 (1972), 697-703. MR0323850(48:2205)

[2] Yu.A. Bahturin, Identical relations in Lie algebras (VNU Science Press, Utrecht, 1987). MR:0886063(88f:17032)

[3] A. Berele, Homogenous polynomial identities, Israel J. Math 42 (1982), 258-272. MR0687131 (84b:16018)

[4] M. Gromov, Groups of polynomial growth and expanding maps, Publ. Math. IHES 53 (1981), 53-73. MR0623534 (83b:53041)

[5] V.G. Kac, Simple irreducible graded Lie algebras of finite growth (in Russian), Math. USSR - Izvestija 2 (1968), 1271-1311. MR0259961 (41:4590)

[6] S. Kobayashi and M. Sanami, Growth of two-step solvable Lie algebras, Proc. Amer. Math. Soc. 109 (1990), no. 4, 859-863. MR1015681 (91d:17011)

[7] A.I. Lichtman, Growth in enveloping algebras, Israel J. Math. 47 (1984), no. 4, 296-304. MR:0764299 (86b:17008)

[8] A.I. Lichtman and V.A. Ufnarovski, On growth of Lie algebras, Algebra Colloq. 2 (1995), no. 1, 45-49. MR,1314454 (95m:17002)

[9] V.M. Petrogradsky, Intermediate growth in Lie algebras and their enveloping algebras, $J$. Algebra 179 (1996), no. 2, 459-482. MR1367858 (96m:17018)

[10] A. Shalev, Simple Lie algebras and Lie algebras of maximal class, Arch. Math. 63 (1994), 297-301. MF 1290602 (95j:17025)

[11] A.L. Shmelkin, Free polynilpotent groups, Soviet Math. Dokl. 7 (1966), 1060-1061.

Department of Mathematics, The University of Western Ontario, London, Ontario, Canada N6A 5B7

E-mail address: dmriley@uwo.ca

Department of Mathematics, The University of Western Ontario, London, Ontario, CANADA N6A 5B7

E-mail address: husefi@uwo.ca 